\title{
OPEN ApoE4 attenuates autophagy via FoxO3a repression in the brain
}

\author{
Hee-Young Sohn ${ }^{1,3}$, Seong-Ik Kim², Jee-Yun Park ${ }^{1}$, Sung-Hye Park ${ }^{2}$, Young Ho Koh ${ }^{1}$, \\ Joon $\mathrm{Kim}^{3 凶}$ \& Chulman Jo ${ }^{1 \bowtie}$
}

Apolipoprotein E (ApoE) plays multiple roles in lipid transport, neuronal signaling, glucose metabolism, mitochondrial function, and inflammation in the brain. It is also associated with neurodegenerative diseases, and its influence differs depending on the isoform. In particular, the $\varepsilon 4$ allele of $A P O E$ is the highest genetic risk factor for developing late-onset Alzheimer's disease (AD). However, the mechanism by which ApoE4 contributes to the pathogenesis of $A D$ remains unclear. We investigated the effect of ApoE4 on autophagy in the human brains of ApoE4 carriers. Compared to non-carriers, the expression of FoxO3a regulating autophagy-related genes was significantly reduced in ApoE4 carriers, and the phosphorylation level of FoxO3a at Ser253 increased in ApoE4 carriers, indicating that FoxO3a is considerably repressed in ApoE4 carriers. As a result, the protein expression of FoxO3a downstream genes, such as Atg12, Beclin-1, BNIP3, and PINK1, was significantly decreased, likely leading to dysfunction of both autophagy and mitophagy in ApoE4 carriers. In addition, phosphorylated tau accumulated more in ApoE4 carriers than in non-carriers. Taken together, our results suggest that ApoE4 might attenuate autophagy via the repression of FoxO3a in $A D$ pathogenesis. The regulation of the ApoE4-FoxO3a axis may provide a novel therapeutic target for the prevention and treatment of $A D$ with the APOE4 allele.

Apolipoprotein E (ApoE) is a multifunctional glycoprotein that plays a major role in redistributing cholesterol and other lipids by binding to cell-surface ApoE receptors such as LDL receptor (LDLR) and LDL receptorrelated protein 1 (LRP1) in the brain ${ }^{1}$. In addition to lipid transport, ApoE modulates multiple pathways in the brain, including synaptic plasticity, glucose metabolism, mitochondrial function, and cerebrovascular function ${ }^{2-4}$. There are three ApoE isoforms (ApoE2, ApoE3, and ApoE4) in humans that have different effects on biological processes ${ }^{5,6}$. Among the three isoforms, the APOE4 allele is associated with an increased risk of Alzheimer's disease (AD) and a lower age of onset ${ }^{7}$. $\mathrm{AD}$ is pathologically characterized by the presence of extracellular amyloid plaques and intracellular neurofibrillary tangles composed of phosphorylated tau protein, as well as extensive neuronal and synaptic losses ${ }^{8,9}$. Growing evidence suggests that the effect of ApoE4 on AD risk is exerted through either the clearance inhibition or aggregation promotion of pathological tau as well as amyloid-beta ${ }^{3,10,11}$; however, the exact etiology of ApoE4 in AD remains elusive.

Macroautophagy (hereafter referred to as autophagy) is a conserved intracellular process that clears aggregated proteins and damaged cellular organelles ${ }^{12}$. Aberrant autophagy contributes to protein aggregation, organelle impairment, and neuronal loss, ultimately leading to neurodegenerative diseases such as AD, Parkinson's disease, and Huntington's disease ${ }^{12,13}$. In particular, the number of autophagic vacuoles, an intermediate doublemembraned vesicle in the autophagy pathway, is higher in the brains of individuals with neurodegenerative diseases than in those of healthy controls suggesting an impairment in the maturation of autophagosomes to autolysosomes ${ }^{4,14}$. Mitochondrial dysfunction and impairment of mitophagy have also been implicated in aging and neurodegenerative diseases such as $\mathrm{AD}^{15,16}$.

Mitophagy, selective autophagy of mitochondria, is an important cellular process for quality control and clearance of mitochondria ${ }^{16}$. Mitophagy is mediated by either the PTEN-induced serine/threonine kinase (PINK1)Parkin pathway or the receptor-mediated pathway ${ }^{16}$. PINK1 senses mitochondrial damage and phosphorylates ubiquitin at Ser65 ${ }^{17}$. P-ubiquitin (Ser65) triggers parkin activation, and then autophagy receptor proteins, including optineurin, NDP52, and p62, are recruited. These proteins mediate the degradation of damaged mitochondria by interacting with the autophagosomal protein LC3 or gamma-aminobutyric acid receptor-associated protein $\left(\right.$ GABARAP) ${ }^{16,18,19}$. Some outer mitochondrial membrane proteins, such as BCL2-interacting protein 3 (BNIP3)

${ }^{1}$ Division of Brain Disease Research, Department for Chronic Disease Convergence Research, Korea National Institute of Health, 187 Osongsaengmyeong2-ro, Osong-eup, Cheongju-si, Chungcheongbuk-do 28159, Republic of Korea. ${ }^{2}$ Department of Pathology, Seoul National University College of Medicine, Seoul 03080, Republic of Korea. ${ }^{3}$ Laboratory of Biochemistry, Division of Life Sciences, Korea University, Seoul 02841, Republic of Korea. ${ }^{\circledR}$ email: joonkim@korea.ac.kr; chulmanjo@gmail.com 
A

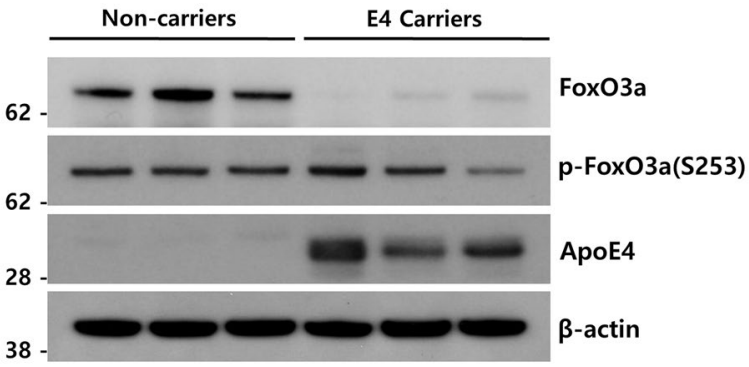

B

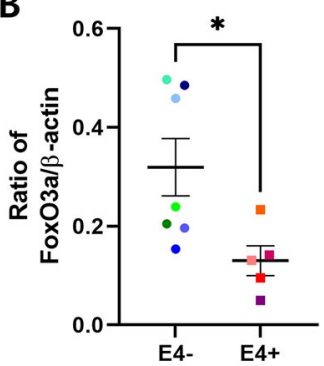

C

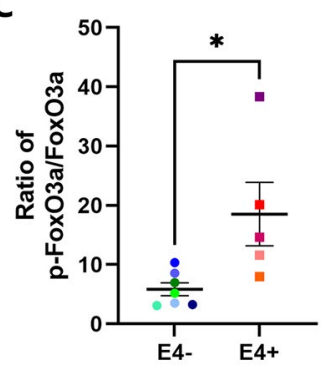

Figure 1. FoxO3a was repressed in human brains of ApoE4 carriers. Human postmortem brain tissues (superior temporal gyrus) were collected to compare ApoE4 non-carriers $(\mathrm{n}=7, \mathrm{E} 4-)$ vs. carriers $(\mathrm{n}=5, \mathrm{E} 4+)$. The protein levels of FoxO3a, p-FoxO3a (Ser253), ApoE4, and $\beta$-actin were analyzed by immunoblotting using the corresponding antibodies, respectively. (A) shows the representative western blot analysis of FoxO3a and p-FoxO3a (Ser253). $\beta$-actin was used as a loading control. Full blots are provided in Supplementary Fig. S4. Relative ratio of FoxO3a (B) and p-FoxO3a (Ser253) (C) to the protein level of $\beta$-actin and FoxO3a, respectively. Data shown are mean \pm SEM and were analyzed using the Student's t-test. $\left({ }^{*} p<0.05\right)$.

and Nip3-like protein X (NIX), promote mitophagy under stress conditions in an independent manner of the PINK1-Parkin pathway ${ }^{16,20}$.

FoxO transcription factors mainly function to maintain cellular and organismal homeostasis, especially in response to stresses such as oxidative and metabolic stress ${ }^{21,22}$. In mammals, there are four FoxOs: FoxO1, FoxO3a, FoxO4, and FoxO6 ${ }^{23}$. Among them, FoxO3a is highly expressed in the brain and controls multiple metabolic pathways for neuronal protection ${ }^{21}$. In addition, FoxO3a is associated with the transcriptional regulation of autophagy in various tissues as well as in adult neural stem cells $\mathrm{s}^{24-26}$. It activates the transcription of several autophagy genes, such as ATG12, VPS34, and BECN1, as well as mitophagy genes BNIP3 and PINK1 ${ }^{24,27}$. FoxO activity is controlled by their subcellular localization, and multiple Ser/Thr kinases such as AKT, serum and glucocorticoid-induced kinase, and AMPK signaling pathways are involved in the translocation of FoxOs ${ }^{23}$. In the regulation of FoxO3a, AKT can phosphorylate FoxO3a at Thr32, Ser253, and Ser315 residues, leading to the inhibition of FoxO3a activity via the docking of 14-3-3 and exclusion of FoxO3a from the nucleus ${ }^{24,28}$.

Several lines of accumulating evidence suggest that FoxO3a positively regulates the expression of several autophagy- and mitophagy-related genes, thus protecting cells against protein toxicity from the aggregation of misfolded or abnormal proteins ${ }^{25,26,29,30}$. In this study, we examined the protein levels of FoxO3a and its downstream genes in the human brain tissues of elderly ApoE4 carriers. We found that the expression levels of FoxO3a and its autophagy- and mitophagy-related downstream genes were significantly lower in ApoE4 carriers than in non-carriers. Thus, our results suggest that ApoE4 plays a crucial role in $\mathrm{AD}$ pathology, such as dysfunction of autophagy and mitophagy through the repression of FoxO3a.

\section{Results}

FoxO3a was repressed in the brains of ApoE4 carriers. Previous studies have reported that ApoE4 is associated with dysfunction of autophagy in the brain, and the transcription factor FoxO3a acts as a regulator of autophagy in multiple cells $\mathrm{s}^{2431,32}$. Based on this, we hypothesized that ApoE4 influences autophagy via FoxO3a. Therefore, we investigated the expression level of FoxO3a protein in human brain tissues of ApoE4 carriers and compared them with those in non-carriers. As shown in Fig. 1A,B, the expression level of FoxO3a protein was significantly lower in ApoE4 carriers than in non-carriers $(p<0.05)$. Intracellular localization is important for FoxO3a transcriptional activity. The phosphorylation of FoxO3a inhibits its transcriptional activity by sequestering it in the cytoplasm ${ }^{23}$. Therefore, we examined the level of phosphorylated FoxO3a at Ser253 between ApoE4 carriers and non-carriers. The level of phosphorylated FoxO3a at Ser253 was dramatically higher in ApoE4 carriers than in non-carriers $(p<0.05)$ (Fig. 1A,C). Together, these results indicate that ApoE4 inhibits the expression and transcriptional activity of FoxO3a. 
A
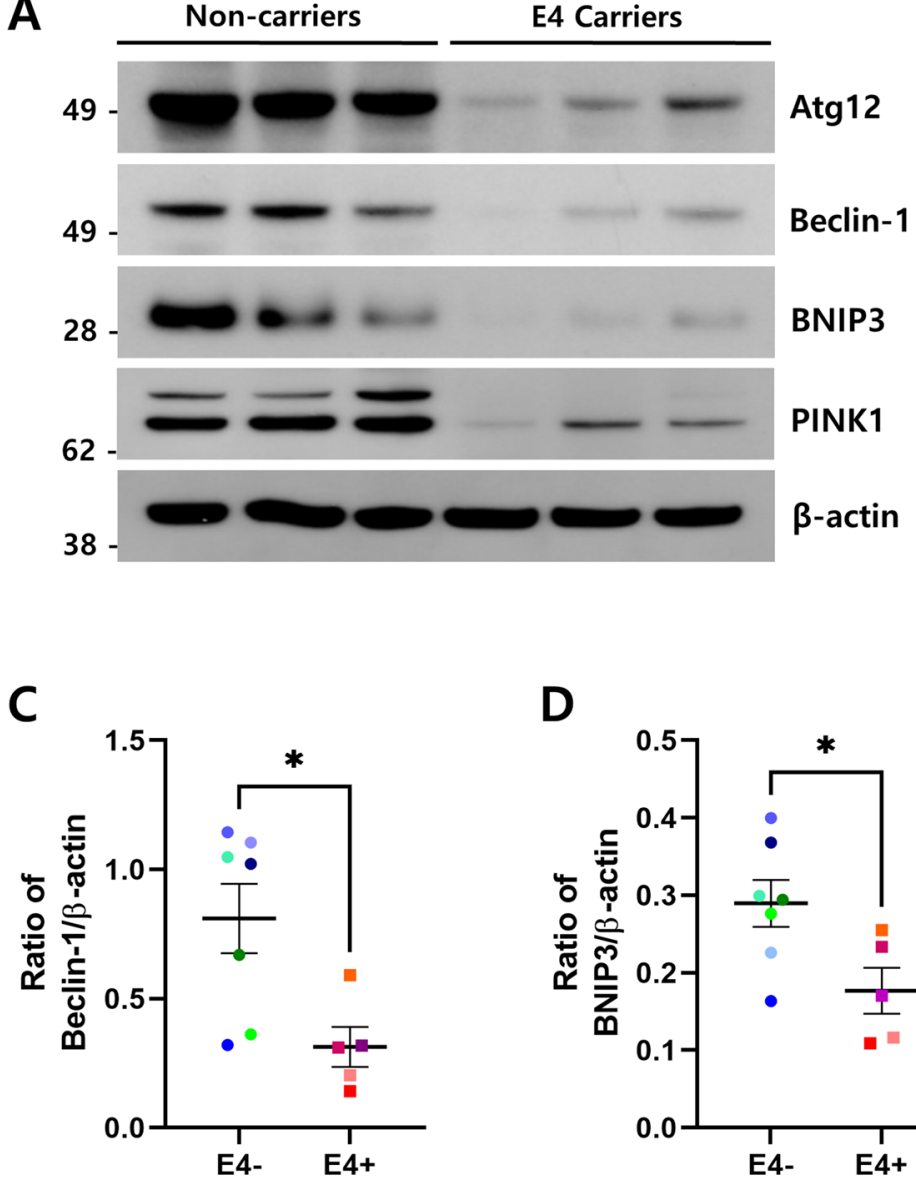

D

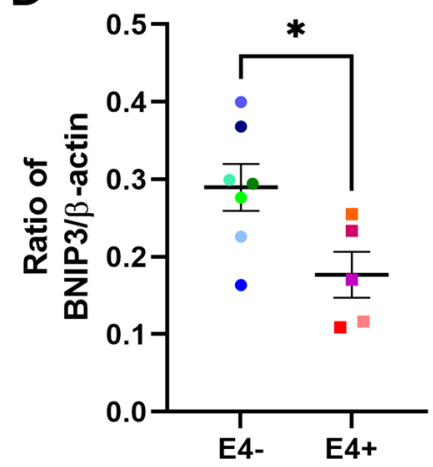

B

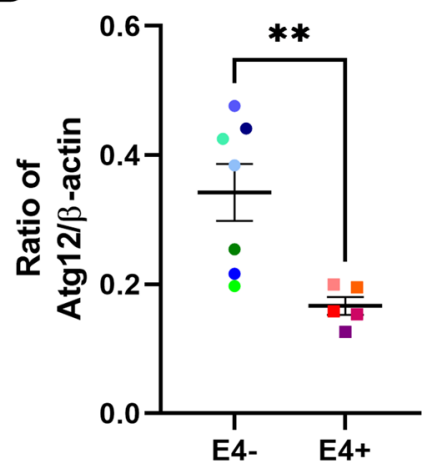

$\mathbf{E}$

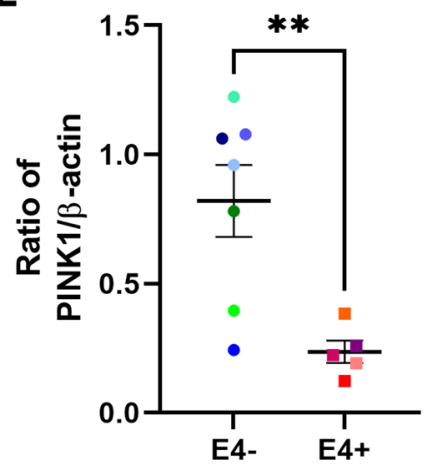

Figure 2. Protein levels of FoxO3a downstream genes decreased in ApoE4 carriers. The protein levels of Atg12, Beclin-1, BNIP3, PINK1, and $\beta$-actin in ApoE4 non-carriers $(n=7, E 4-)$ vs. carriers $(n=5, E 4+)$ were analyzed by immunoblotting using the corresponding antibodies, respectively. (A) shows the representative western blot analysis of Atg12, Beclin-1, BNIP3, and PINK1. $\beta$-actin was used as a loading control. Full blots are provided in Supplementary Fig. S4. Relative ratio of Atg12 (B), Beclin-1 (C), BNIP3 (D) and PINK1 (E) against the protein level of $\beta$-actin. Data shown are mean \pm SEM and were analyzed using the Student's t-test. $\left({ }^{*} p<0.05,{ }^{* *} p<0.01\right)$.

Autophagy-related gene products decreased in ApoE4 carriers. We further tested whether the repression of FoxO3a affects the expression of downstream genes related to autophagy and mitophagy. The expression levels of Atg12, Beclin-1, BNIP3, and PINK1 proteins were analyzed by immunoblotting (Fig. 2A). Compared with non-carriers, the expression levels of Atg12, Beclin-1, BNIP3, and PINK1 proteins were significantly reduced in ApoE4 carriers (Fig. 2B-E). To confirm whether the decrease in these proteins was caused by the suppression of FoxO3a activity, we conducted correlation analyses for the levels of FoxO3a and Atg12, Beclin-1, BNIP3, and PINK1. Positive correlations were observed in all subjects (Supplementary Fig. S2). Together, our data suggest that ApoE4 significantly decreases autophagy/mitophagy component proteins via the repression of FoxO3a, which likely leads to the alteration of both autophagy and mitophagy.

Autophagy/mitophagy were dysregulated in ApoE4 carriers. To further examine the autophagic status, we analyzed the expression levels of autophagy markers LC3 and p62 (Fig. 3A). The ratio of LC3-II/LC3-I was increased in the brains of ApoE4 carriers compared with non-carriers (Fig. 3D). Although the conversion ratio of LC3-I to LC3-II is used as a measure of autophagy activation, an increase in autophagosomes has been observed in the brains of $\mathrm{AD}$ patients through autophagy dysfunction ${ }^{33}$. This suggests that the impairment of maturation in autophagosome to lysosome fusion could induce the accumulation of autophagosomes with LC3II, thus increasing the autophagosomal marker LC3-II. In addition, p62 protein levels also increased (Fig. 3C). Because p62 itself is degraded during autophagy, it is speculated that the increase of p62 protein along with LC3II might be resulted from autophagy dysregulation in ApoE4 carriers.

To assess the status of mitophagy in the brains of ApoE4 carriers, we examined the expression levels of p-ubiquitin, which is a substrate of PINK1, and optineurin, a well-known mitophagy receptor protein, by immunoblotting (Fig. 3B). As shown in Fig. 3E, the phosphorylation level of the mitophagy activation marker ubiquitin was significantly reduced in ApoE4 carriers compared with non-carriers $(p<0.01)$, indicating that the activity of PINK1 decreased in ApoE4 carriers. In addition, the level of the autophagy receptor optineurin also decreased in ApoE4 carriers (Fig. 3F). The reduction of p-ubiquitin and optineurin indicated that the mitophagy pathway 
A

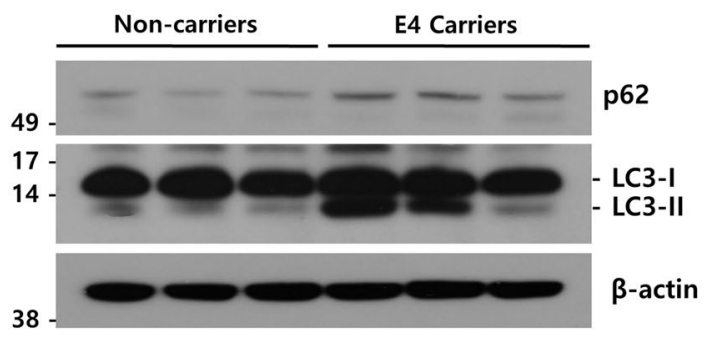

C

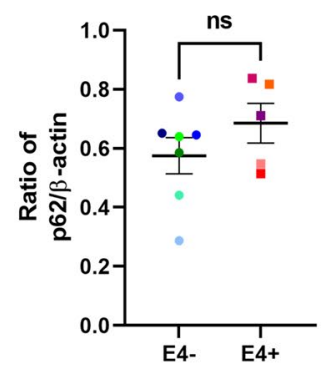

E

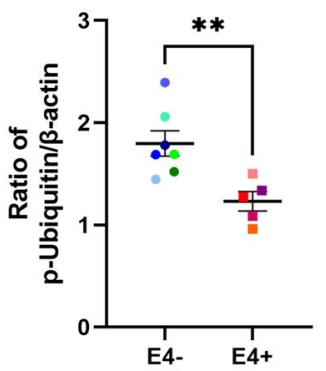

D

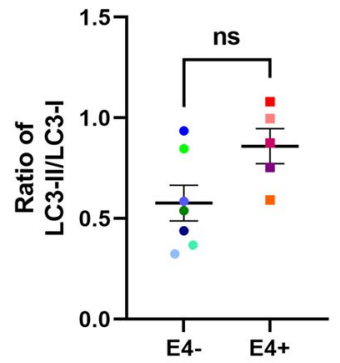

$\mathbf{F}$

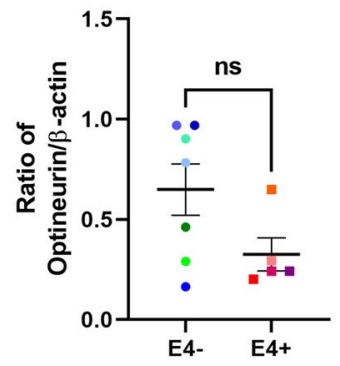

Figure 3. Autophagy/mitophagy components were quantitatively altered in the brains of ApoE4 carriers. The protein levels of p62, LC3, p-ubiquitin (Ser65), optineurin, and $\beta$-actin in ApoE4 non-carriers $(n=7$, E4-) vs. carriers $(n=5, E 4+)$ were analyzed by immunoblotting using corresponding antibodies, respectively. Representative western blot analysis of p62 and LC3 (A), p-ubiquitin (Ser65), and optineurin (B). $\beta$-actin was used as a loading control. Full blots are provided in Supplementary Fig. S4. Relative ratio of p62 (C), LC3-II (D) p-ubiquitin (Ser65) (E), and optineurin (F) against the protein levels indicated on the $y$-axis, respectively. Data shown are mean \pm SEM. $\left({ }^{* *} p<0.01\right)$. ns not significant.

was also suppressed in ApoE4 carriers. Together, these data suggest that ApoE4 might influence the dysfunction of both autophagy and mitophagy via the repression of FoxO3a.

Accumulation of phosphorylated tau increased in ApoE4 carriers. To examine the effect of FoxO3a reduction on tau pathology in ApoE4 carriers, we measured the levels of phosphorylated tau and total tau proteins by immunoblotting (Fig. 4A). Among the 12 cases, one case of ApoE4 carriers was a vascular dementia (VD) patient. Because the pathology of patients with VD is largely different from that of $\mathrm{AD}$, it was excluded from the quantification and correlation analysis of tau pathology. As shown in Fig. 4B, the levels of tau phosphorylated at Ser396/404 epitopes detected by a PHF1 antibody were significantly increased in ApoE4 carriers compared with non-carriers $(p<0.05)$. Moreover, the level of tau phosphorylated at Ser262 also increased (Fig. 4C). In contrast, the expression level of total tau protein was lower in ApoE4 carriers compare to non-carriers $(p<0.01$; Fig. $4 \mathrm{D})$. Interestingly, the Braak stage, which indicates the degree of tau pathology, was higher in ApoE4 carriers than in non-carriers compared to AD patients of similar age (Supplementary Table). Together, these results suggest that ApoE4 may accelerate the progression of tau pathophysiology. Furthermore, we performed a correlation analysis between the levels of FoxO3a and phosphorylated tau proteins. As shown in Fig. 5A, the level of phosphorylated tau at Ser396/404 residues was negatively correlated with that of FoxO3a $\left(\mathrm{R}^{2}=0.3127 ; p=0.0737 ; \mathrm{n}=11\right)$. The level of phosphorylated tau at Ser262 also showed a negative, significant correlation with that of FoxO3a $\left(\mathrm{R}^{2}=0.3855 ; p=0.0415 ; \mathrm{n}=11\right.$; Fig. $\left.5 \mathrm{~B}\right)$. These results demonstrate that the repression of FoxO3a might contribute to the accumulation of phosphorylated tau proteins in ApoE4 carriers.

\section{Discussion}

Proteostasis is a process of protein homeostasis that maintains the quality control of proteins and organelles from synthesis to degradation, and autophagy is an intracellular degradation pathway that removes misfolded and proteotoxic proteins ${ }^{34,35}$. Impaired autophagy in the brain is a common feature of aging and neurodegenerative diseases ${ }^{36,37}$. Therefore, modulation of autophagy is important for preventing the occurrence and progression of neurodegenerative diseases. In this study, we showed that ApoE4 represses FoxO3a, resulting in decreased protein expression of autophagy- and mitophagy-related genes that are regulated by FoxO3a. We suggest that the reduction of these autophagy-essential proteins might induce dysfunction of autophagy and mitophagy and subsequently lead to an increase in phosphorylated tau proteins in ApoE4 carriers (Fig. 6). A previous study observed that serum FoxO3a was significantly lower in $\mathrm{AD}$ than in mild cognitive impairment and geriatric control ${ }^{38}$. Moreover, it was found that mitochondrial biogenesis, dynamics, and antioxidant response proteins were downregulated in ApoE4 carriers relative to non-carriers, and the level of FoxO3a was lowered in 
A
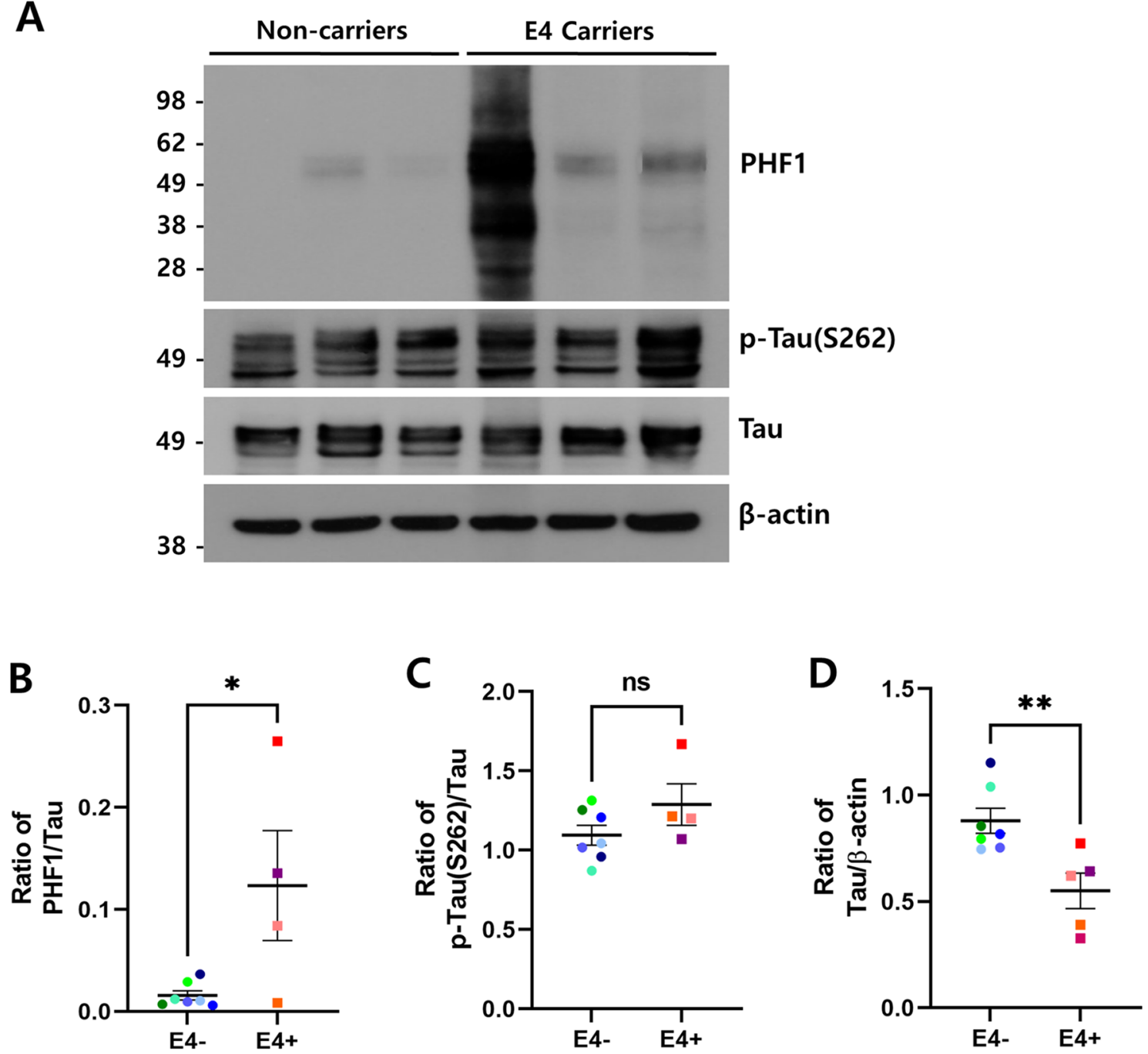

Figure 4. Phosphorylated tau increased in the brain of ApoE4 carriers. The protein levels of phosphorylated tau, total tau, and $\beta$-actin in ApoE4 non-carriers $(n=7, E 4-)$ vs. carriers $(n=5, E 4+)$ were analyzed by immunoblotting using the corresponding antibodies, respectively. (A) shows the representative western blot analysis of tau phosphorylated at Ser396/404 (PHF1) and Ser262 (p-tau [S262]) and total tau proteins. $\beta$-actin was used as a loading control. Full blots are provided in Supplementary Fig. S4. Relative ratio of tau phosphorylated at Ser396/404 (B) and Ser262 (C), and total tau (D) to the protein levels of tau and $\beta$-actin, respectively. All data are presented as mean \pm SEM. $\left({ }^{\star} p<0.05,{ }^{* *} p<0.01\right) . n s$ not significant.

postmortem human brains ${ }^{39}$. These findings are consistent with our work showing that the repression of FoxO3a by ApoE4 might be involved in AD pathology.

ApoE4 is involved in autophagy dysfunction ${ }^{31,32}$. Simonovitch et al. demonstrated that autophagy is impaired in ApoE4 astrocytes ${ }^{31}$. Furthermore, a recent study suggested that ApoE4 inhibits the expression of autophagyrelated genes by competitively binding ApoE4 to the coordinated lysosomal expression and regulation (CLEAR) DNA elements in which transcription factor EB (TFEB), a master regulator of autophagy and lysosome biogenesis, normally induces its downstream genes ${ }^{32}$. Here, we added FoxO3a as an intermediator in ApoE4-mediated autophagy dysfunction. As shown in Fig. 1, ApoE4 strongly represses the expression of FoxO3a protein. Therefore, it is speculated that ApoE4 inhibits FoxO3a expression especially in the transcription level. Therefore, we analyzed whether the CLEAR element is in FoxO3a promoter region. We found a CLEAR element in the promoter region of human FoxO3a (data not shown). Thus, it is possible that ApoE4 competitively inhibits the binding of TFEB to the CLEAR element in the promoter of FoxO3a, thus repressing the expression of FoxO3a. In addition, we examined the association between ApoE and FoxO3a using the co-immunoprecipitation technique. Interestingly, the amount of FoxO3a co-immunoprecipitated with ApoE4 was increased than that with ApoE3 (Supplementary Fig. S3). Thus, how this interaction could affect the repression of FoxO3a remains to be investigated.

ApoE4 is known to be associated with tau pathology in $\mathrm{AD}^{10,40,41}$. Shi et al. demonstrated that ApoE4 promotes tau pathology, neuroinflammation, and tau-mediated neurodegeneration relative to other ApoE isoforms ${ }^{10}$. Kang et al. reported that ApoE4 exacerbates tau pathology via inhibition of vesicular monoamine transporter ${ }_{2}$ 

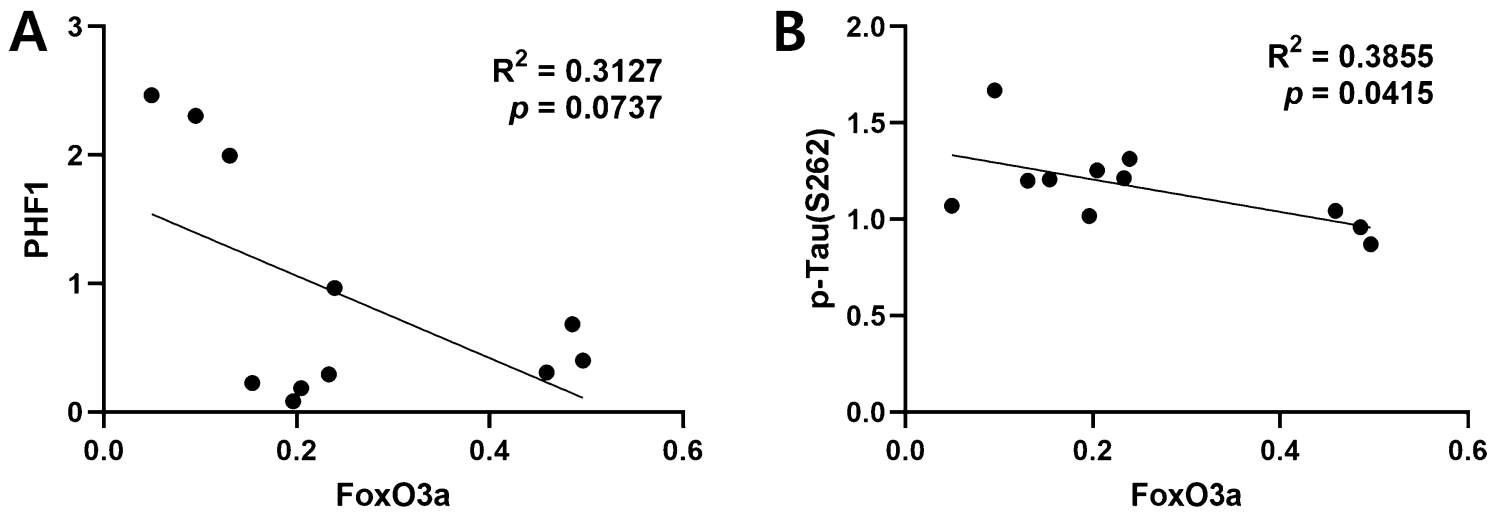

Figure 5. Protein levels of phosphorylated tau were negatively correlated with those of FoxO3a. (A) The correlation of protein levels between FoxO3a and tau phosphorylated at S396/404 (PHF1) was analyzed with the Pearson's correlation test (E4 carriers $n=4$, non-carriers $n=7)$. (B) The correlation of protein levels between FoxO3a and tau phosphorylated at S262 protein levels was analyzed with the Pearson's correlation test (E4 carriers $n=4$, non-carriers $n=7$ ). Linear regressions are shown as solid lines.

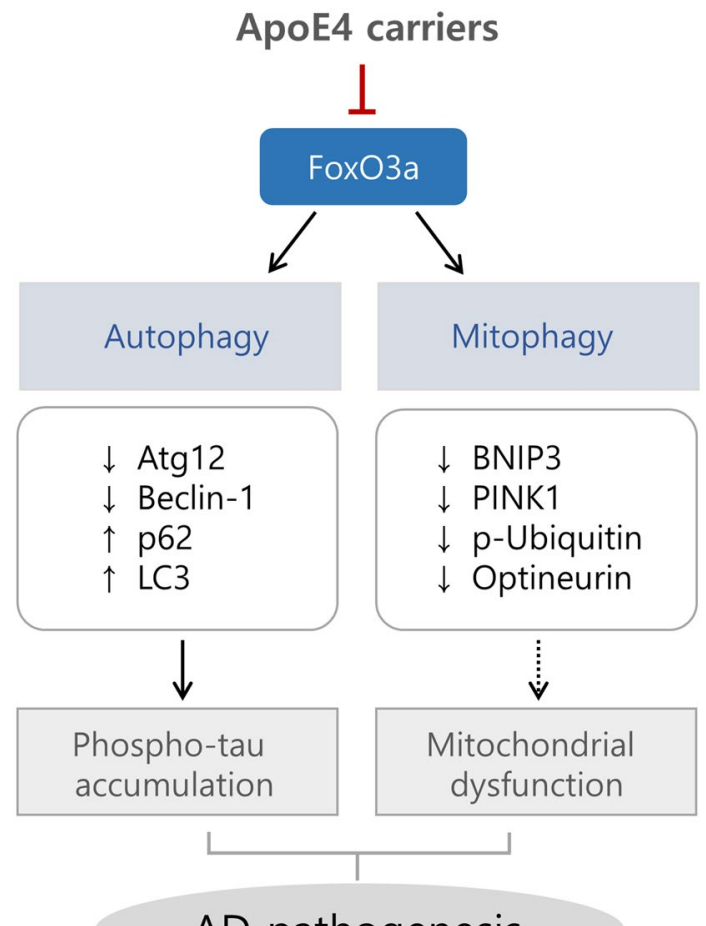

\section{AD pathogenesis}

Figure 6. Schematic diagram of the relationship between ApoE4 and FoxO3a in AD pathogenesis. ApoE4 represses the transcription factor FoxO3a. Downregulation of FoxO3a reduces the protein levels of its autophagy/mitophagy-related downstream genes, such as Atg12, Beclin-1, BNIP3, and PINK1, which could result in an impairment of autophagy and mitophagy. Dysfunction of autophagy might increase the levels of phosphorylated tau proteins, leading to $\mathrm{AD}$ pathogenesis.

$\left(\mathrm{VMAT}_{2}\right)$ in the locus coeruleus, reduces hippocampal volume, and induces cognitive dysfunction in $\mathrm{AD}^{40}$. Another study suggested that human iPSC-derived human ApoE4-expressing neurons show higher levels of tau phosphorylation than those expressing human ApoE3 in mouse models ${ }^{41}$. Thus, these results are in line with ours: ApoE4 worsens tau pathology. In contrast, Zhao et al. suggested that ApoE2 is associated with increased tau pathology relative to ApoE3 or ApoE4 in primary tauopathies such as progressive supranuclear palsy and corticobasal degeneration ${ }^{42}$. Therefore, the relevance of ApoE isoforms in tauopathy requires further investigation.

ApoE4 is associated with mitochondrial dysfunction, and impaired mitophagy may contribute to mitochondrial dysfunction and lead to AD pathogenesis ${ }^{39,43,44}$. However, the molecular mechanism by which ApoE4 impairs mitochondrial function is not well understood. Here, we observed decreased expression of BNIP3 and 
PINK1, the key molecules in mitophagy, in ApoE4 carriers compared to ApoE4 non-carriers (Fig. 2D,E). In addition, the phosphorylation level of ubiquitin at Ser65, which is known to be directly phosphorylated by PINK $1^{17}$, was significantly reduced (Fig. 3B,E), suggesting that the activity of PINK1 is highly suppressed in ApoE4 carriers. Thus, our results explain for the first time how ApoE4 evokes mitochondrial and mitophagic dysfunction in $\mathrm{AD}$ brains with the APOE4 allele.

Mitochondrial dysfunction has been strongly implicated in glucose hypometabolism, represented by compromised energy metabolism in the early stages of AD pathology ${ }^{15,45}$. Glucose hypometabolism in the brain is associated with impairment of insulin signaling as well as cognitive decline ${ }^{15,45}$. FDG-PET neuroimaging studies have also shown that cerebral glucose metabolism is reduced in regions associated with $\mathrm{AD}$, especially in ApoE4 homozygote individuals ${ }^{46,47}$. A study using human ApoE4-targeted replacement mice revealed that ApoE4 interferes with neuronal insulin signaling by trapping insulin receptors in the endosomes ${ }^{48}$, explaining how ApoE4 affects the reduction of glucose metabolism in the brain. FoxOs have been shown to play an important role in upregulating genes such as glucose 6 phosphatase (G6Pase) and phosphoenolpyruvate (PEPCK), which control glucose metabolism ${ }^{49}$. Considering the result that ApoE4 represses FoxO3a (Fig. 1), ApoE4 could reduce glucose metabolism via FoxO3a in the brain. In addition, mitochondrial dysfunction could be elicited by ApoE4mediated downregulation of mitophagy genes such as BNIP3 and PINK1via FoxO3a repression (Fig. 2D,E). Thus, it is inferred that ApoE4 could result in glucose hypometabolism in the brain via various metabolic processes, such as FoxO3a repression and insulin signaling interference.

FoxO3a maintains neural stem cell homeostasis as well as mature neurons. FoxO3a knockout neural stem cells (NSCs) show decreased self-renewal, increased apoptosis, increased oxidative metabolism, and depletion of the NSC pool ${ }^{50,51}$. In addition, FoxO3a directly binds genes involved in the autophagy network through genomic approaches and modulates the induction of autophagy in neural stem and progenitor cell ${ }^{26}$. These findings suggest that FoxO3a is likely to be critical for maintaining neuronal homeostasis during neuronal development. Conversely, defective FoxO3a may induce an imbalance in the neuronal network, eventually leading to neurodegenerative diseases such as AD. According to recent studies, KLOTHO-VS heterozygosity is associated with reduced $\mathrm{AD}$ risk and $\beta$-amyloid burden in individuals carrying the APOE4 allele ${ }^{52,53}$. However, it remains unclear how it biologically protects ApoE4 carriers from AD. A previous study showed that Klotho reduces oxidative stress by negatively regulating the PI3K/AKT signaling pathway and subsequently enhancing FoxO3a-mediated MnSOD expression in the kidneys of mouse models ${ }^{54}$. In addition, several meta-analyses have shown that KLOTHO-VS is significantly associated with exceptional longevity (aged $85+$ ) along with APOE and FOXO3A $A^{55,56}$. Thus, FoxO3a may be a key player in the health and age-related diseases of the brain.

Our study has some limitations. First, there were no APOE4 homozygote tissues in ApoE4 carriers. If the experiments, including APOE4 homozygous samples, had been conducted, the differences in biological processes according to ApoE isoforms could have been more clearly observed. In addition, as the number of ApoE4 carriers was insufficient, comparisons could not be made with the same number of non-carriers. Second, we do not know the biological mechanism by which ApoE4 reduces FoxO3a expression although we have demonstrated an increased interaction between these proteins. Therefore, further studies are needed to elucidate the mechanism by which ApoE4 regulates FoxO3a.

In conclusion, we suggest that ApoE4 may contribute to $\mathrm{AD}$ pathogenesis by attenuating autophagy and mitophagy through the repression of FoxO3a. The APOE and FOXO3A genes have been consistently reported by genome-wide association studies as the two most robust genetic loci associated with longevity across populations ${ }^{57,58}$. However, there was no link between ApoE and FoxO3a in biological processes. Herein, we identified a relationship between ApoE4 and FoxO3a on autophagy in the brain for the first time. Our findings may serve as a novel therapeutic strategy for ApoE4 carriers in AD.

\section{Methods}

Ethic statement. All participants were recruited from the Seoul National University Hospital Brain Bank (SNUHBB). In all cases, written informed consent for the research was obtained from the patient before death or legal guardian. This study was approved by the Institutional Review Board of the Korea Disease Control and Prevention Agency for using human brain tissue samples (IRB number: 2019-05-02). All experiments were performed following relevant guidelines and regulations.

Human brain tissue. Human brain tissues were provided from the SNUHBB, where neuropathological diagnosis of the postmortem human brain was performed. The diagnosis of $\mathrm{AD}$ followed the diagnostic criteria of the National Institute of Aging and Alzheimer's Association (NIA-AA) guidelines ${ }^{59}$. The brain tissues of AD patients used in this study were all positive for amyloid and phospho-tau deposition (Supplementary Fig. S1 and Table). Five ApoE4 carriers and seven non-carriers were enrolled in this study. The patient's epidemiological information was summarized in the Supplementary Table.

Antibodies. Anti-FoxO3a (2497), phospho-FoxO3a (Ser253) (13129), Atg12 (4280), Beclin-1 (3495), BNIP3 (44060), PINK1 (6946), LC3 (12741), p62/SQSTM1 (8025), phospho-ubiquitin (Ser65) (62802), and optineurin (58981) antibodies were purchased from Cell Signaling Technology. Anti-ApoE4 (MABN43) and $\beta$-actin (A5316) antibodies were obtained from Sigma-Aldrich. Anti-tau (A0024) and phospho-tau (Ser262) antibodies were purchased from DAKO and Abcam, respectively. Anti-PHF1 antibody for detecting phosphorylated tau at Ser396/404 residues was described in a previous study ${ }^{60}$.

Immunoblotting. Human brain tissues (superior frontal gyrus, $200 \mathrm{mg}$ ) were homogenized and lysed in $500 \mu \mathrm{l}$ of RIPA buffer (Cell Signaling Technology) containing $1 \mathrm{mM} \mathrm{NaF}$ and $1 \times$ protease inhibitor cocktail 
(Sigma-Aldrich) on ice. Tissue lysates were harvested by centrifugation at $13,000 \mathrm{rpm}$ for $30 \mathrm{~min}$ at $4{ }^{\circ} \mathrm{C}$, and the protein concentration was determined using the Bradford assay (Bio-Rad). Total proteins were separated by NuPAGE 4-12\% Bis-Tris Gels (Thermo Fisher Scientific) in MES SDS running buffer (Thermo Fisher Scientific) and then transferred to PVDF membranes (Millipore). The membranes were blocked with $5 \%$ skim milk in Trisbuffered saline with $0.1 \%$ tween-20 for $1 \mathrm{~h}$ and probed with specific antibodies at $4{ }^{\circ} \mathrm{C}$ overnight. Immune complexes were detected using horseradish peroxidase-conjugated anti-rabbit or anti-mouse antibodies, followed by enhanced chemiluminescence (ECL, Amersham). For the re-blotting, the membrane was stripped using the Restore Western Blot Stripping Buffer (Thermo Fisher Scientific, 21059) and then incubated with an antibody of interest. The densitometric analyses of the western blots were performed using NIH ImageJ software.

Statistical analysis. All statistical analyses were performed using GraphPad Prism software (version 9.1.0). $P$-values were calculated using a two-tailed Student's t-test. Pearson's correlation analysis was used to measure the degree of association between the two variables. A $p$-value of less than 0.05 was considered statistically significant.

Received: 3 June 2021; Accepted: 19 August 2021

Published online: 02 September 2021

\section{References}

1. Holtzman, D. M., Herz, J. \& Bu, G. Apolipoprotein E and apolipoprotein E receptors: Normal biology and roles in Alzheimer disease. Cold Spring Harbor Perspect. Med. 2, a0006312 (2012).

2. Tudorache, I. F., Trusca, V. G. \& Gafencu, A. V. Apolipoprotein E-a multifunctional protein with implications in various pathologies as a result of its structural features. Comput. Struct. Biotechnol. J. 15, 359-365 (2017).

3. Yamazaki, Y., Zhao, N., Caulfield, T. R., Liu, C.-C. \& Bu, G. Apolipoprotein E and Alzheimer disease: Pathobiology and targeting strategies. Nat. Rev. Neurol. 15, 501-518 (2019).

4. Uddin, M. S. et al. APOE and Alzheimer's disease: Evidence mounts that targeting APOE4 may combat Alzheimer's pathogenesis. Mol. Neurobiol. 56, 2450-2465 (2019).

5. Zannis, V. I. et al. Proposed nomenclature of apoE isoproteins, apoE genotypes, and phenotypes. J. Lipid Res. 23, $911-914$ (1982).

6. Yin, Y. \& Wang, Z. ApoE and neurodegenerative diseases in aging. Aging Aging Relat. Dis. 2, 77-92 (2018).

7. Saunders, A. M. et al. Association of apolipoprotein E allele $€ 4$ with late-onset familial and sporadic Alzheimer's disease. Neurology 43, 1467-1467 (1993).

8. Long, J. M. \& Holtzman, D. M. Alzheimer disease: an update on pathobiology and treatment strategies. Cell 179, 312-339 (2019).

9. Hanseeuw, B. J. et al. Association of amyloid and tau with cognition in preclinical Alzheimer disease: A longitudinal study. JAMA Neurol. 76, 915-924 (2019).

10. Shi, Y. et al. ApoE4 markedly exacerbates tau-mediated neurodegeneration in a mouse model of tauopathy. Nature 549, 523-527 (2017).

11. Brecht, W. J. et al. Neuron-specific apolipoprotein e4 proteolysis is associated with increased tau phosphorylation in brains of transgenic mice. J. Neurosci. 24, 2527-2534 (2004).

12. Fujikake, N., Shin, M. \& Shimizu, S. Association between autophagy and neurodegenerative diseases. Front. Neurosci. 12, 255 (2018).

13. Nah, J., Yuan, J. \& Jung, Y.-K. Autophagy in neurodegenerative diseases: From mechanism to therapeutic approach. Mol. Cells 38, $381(2015)$

14. Guo, F., Liu, X., Cai, H. \& Le, W. Autophagy in neurodegenerative diseases: Pathogenesis and therapy. Brain Pathol. 28, 3-13 (2018).

15. Perez Ortiz, J. M. \& Swerdlow, R. H. Mitochondrial dysfunction in Alzheimer's disease: Role in pathogenesis and novel therapeutic opportunities. Br. J. Pharmacol. 176, 3489-3507 (2019).

16. Chakravorty, A., Jetto, C. T. \& Manjithaya, R. Dysfunctional mitochondria and mitophagy as drivers of Alzheimer's disease pathogenesis. Front. Aging Neurosci. 11, 311 (2019).

17. Kondapalli, C. et al. PINK1 is activated by mitochondrial membrane potential depolarization and stimulates Parkin E3 ligase activity by phosphorylating Serine 65. Open Biol. 2, 120080 (2012).

18. Bingol, B. \& Sheng, M. Mechanisms of mitophagy: PINK1, Parkin, USP30 and beyond. Free Radic. Biol. Med. 100, 210-222 (2016).

19. Nguyen, T. N., Padman, B. S. \& Lazarou, M. Deciphering the molecular signals of PINK1/Parkin mitophagy. Trends Cell Biol. 26, 733-744 (2016).

20. Rodger, C. E., McWilliams, T. G. \& Ganley, I. G. Mammalian mitophagy-from in vitro molecules to in vivo models. FEBS J. 285, 1185-1202 (2018).

21. Morris, B. J., Willcox, D. C., Donlon, T. A. \& Willcox, B. J. FOXO3: A major gene for human longevity-a mini-review. Gerontology 61, 515-525 (2015).

22. Murtaza, G. et al. FOXO transcriptional factors and long-term living. Oxid. Med. Cell. Longe. 2017, 2 (2017).

23. Greer, E. L. \& Brunet, A. FOXO transcription factors at the interface between longevity and tumor suppression. Oncogene 24, 7410-7425 (2005).

24. Cheng, Z. The FoxO-autophagy axis in health and disease. Trends Endocrinol. Metab. 30, 658-671 (2019).

25. Mammucari, C. et al. FoxO3 controls autophagy in skeletal muscle in vivo. Cell Metab. 6, 458-471 (2007).

26. Audesse, A. J. et al. FOXO3 directly regulates an autophagy network to functionally regulate proteostasis in adult neural stem cells. PLoS Genet. 15, e1008097 (2019).

27. Eijkelenboom, A. \& Burgering, B. M. FOXOs: Signalling integrators for homeostasis maintenance. Nat. Rev. Mol. Cell Biol. 14, 83-97 (2013).

28. Tzivion, G., Dobson, M. \& Ramakrishnan, G. FoxO transcription factors; Regulation by AKT and 14-3-3 proteins. Biochim. Biophys. Acta Mol. Cell Res. 1813, 1938-1945 (2011).

29. Zhao, J. et al. FoxO3 coordinately activates protein degradation by the autophagic/lysosomal and proteasomal pathways in atrophying muscle cells. Cell Metab. 6, 472-483 (2007).

30. Mei, Y. et al. FOXO3a-dependent regulation of Pink1 (Park6) mediates survival signaling in response to cytokine deprivation. Proc. Natl. Acad. Sci. 106, 5153-5158 (2009).

31. Simonovitch, S. et al. Impaired autophagy in APOE4 astrocytes. J. Alzheimers Dis. 51, 915-927 (2016).

32. Parcon, P. A. et al. Apolipoprotein E4 inhibits autophagy gene products through direct, specific binding to CLEAR motifs. Alzheimers Dement. 14, 230-242 (2018). 
33. Long, Z. et al. Dynamic changes of autophagic flux induced by Abeta in the brain of postmortem Alzheimers disease patients, animal models and cell models. Aging 12, 10912 (2020).

34. Kurtishi, A., Rosen, B., Patil, K. S., Alves, G. W. \& Møller, S. G. Cellular proteostasis in neurodegeneration. Mol. Neurobiol. 56, 3676-3689 (2019).

35. Höhn, A., Tramutola, A. \& Cascella, R. Proteostasis failure in neurodegenerative diseases: Focus on oxidative stress. Oxid. Med. Cell. Longe. 2020, 2 (2020).

36. Nixon, R. A. The role of autophagy in neurodegenerative disease. Nat. Med. 19, 983-997 (2013).

37. Barbosa, M. C., Grosso, R. A. \& Fader, C. M. Hallmarks of aging: An autophagic perspective. Front. Endocrinol. 9, 790 (2019).

38. Pradhan, R. et al. Serum FOXO3A: A ray of hope for early diagnosis of Alzheimer's disease. Mech. Ageing Dev. 190, 111290 (2020).

39. Yin, J. et al. Effect of ApoE isoforms on mitochondria in Alzheimer disease. Neurology 94, e2404-e2411 (2020).

40. Kang, S. S. et al. ApoE4 inhibition of VMAT2 in the locus coeruleus exacerbates Tau pathology in Alzheimer's disease. Acta Neuropathol. 2, 1-20 (2021).

41. Wang, C. et al. Gain of toxic apolipoprotein E4 effects in human iPSC-derived neurons is ameliorated by a small-molecule structure corrector. Nat. Med. 24, 647-657 (2018).

42. Zhao, N. et al. APOE $\varepsilon 2$ is associated with increased tau pathology in primary tauopathy. Nat. Commun. 9, 1-11 (2018).

43. Schmukler, E. et al. Altered mitochondrial dynamics and function in APOE4-expressing astrocytes. Cell Death Dis. 11, 1-13 (2020).

44. Fernandez, C. G., Hamby, M. E., McReynolds, M. L. \& Ray, W. J. The role of APOE4 in disrupting the homeostatic functions of astrocytes and microglia in aging and Alzheimer's disease. Front. Aging Neurosci. 11, 14 (2019).

45. Kapogiannis, D. \& Mattson, M. P. Disrupted energy metabolism and neuronal circuit dysfunction in cognitive impairment and Alzheimer's disease. Lancet Neurol. 10, 187-198 (2011).

46. Jack, C. R. Jr. et al. Tracking pathophysiological processes in Alzheimer's disease: An updated hypothetical model of dynamic biomarkers. Lancet Neurol. 12, 207-216 (2013).

47. Ryu, J., Zimmer, E., Rosa-Neto, P. \& Yoon, S. Consequences of metabolic disruption in Alzheimer's disease pathology. Neurotherapeutics 16, 600-610 (2019).

48. Zhao, N. et al. Apolipoprotein E4 impairs neuronal insulin signaling by trapping insulin receptor in the endosomes. Neuron 96, 115-129 (2017).

49. Lee, S. \& Dong, H. H. FoxO integration of insulin signaling with glucose and lipid metabolism. J. Endocrinol. 233, R67 (2017).

50. Renault, V. M. et al. FoxO3 regulates neural stem cell homeostasis. Cell Stem Cell 5, 527-539 (2009).

51. Santo, E. E. \& Paik, J. FOXO in neural cells and diseases of the nervous system. Curr. Top. Dev. Biol. 127, 105-118 (2018).

52. Belloy, M. E., Napolioni, V., Han, S. S., Le Guen, Y. \& Greicius, M. D. Association of klotho-vs heterozygosity with risk of Alzheimer disease in individuals who carry APOE4. JAMA Neurol. 77, 849-862 (2020).

53. Erickson, C. M. et al. KLOTHO heterozygosity attenuates APOE4-related amyloid burden in preclinical AD. Neurology 92, e1878e1889 (2019).

54. Lim, S. W. et al. Klotho enhances FoxO3-mediated manganese superoxide dismutase expression by negatively regulating PI3K/ AKT pathway during tacrolimus-induced oxidative stress. Cell Death Dis. 8, e2972-e2972 (2017).

55. Revelas, M. et al. Review and meta-analysis of genetic polymorphisms associated with exceptional human longevity. Mech. Ageing Dev. 175, 24-34 (2018).

56. Zhu, Z. et al. Klotho gene polymorphisms are associated with healthy aging and longevity: Evidence from a meta-analysis. Mech. Ageing Dev. 178, 33-40 (2019).

57. Broer, L. et al. GWAS of longevity in CHARGE consortium confirms APOE and FOXO3 candidacy. J. Gerontol. Ser. Biomed. Sci. Med. Sci. 70, 110-118 (2015).

58. Morris, B. J., Willcox, B. J. \& Donlon, T. A. Genetic and epigenetic regulation of human aging and longevity. Biochim. Biophys. Acta Mol. Basis Dis. 1865, 1718-1744 (2019).

59. Hyman, B. T. et al. National Institute on Aging-Alzheimer's Association guidelines for the neuropathologic assessment of Alzheimer's disease. Alzheimers Dement. 8, 1-13 (2012).

60. Jo, C. et al. Nrf2 reduces levels of phosphorylated tau protein by inducing autophagy adaptor protein NDP52. Nat. Commun. 5 , $1-13(2014)$.

\section{Acknowledgements}

We thank Drs. Gail VW Johnson (University of Rochester, USA), Michael Greenberg (Harvard Medical School, USA) and Peter Davies (Albert Einstein College of Medicine, USA) for providing materials for this study. This research was supported by a fund (2019-NI-086-02) from Research of Korea Disease Control and Prevention Agency.

\section{Author contributions}

H.S., J.K. and C.J. contributed to the study concept and design. H.S. mainly performed the experiments, analysis, and interpretation of data. H.S. and C.J. wrote the manuscript. J.P. discussed and interpreted data. S.K. and S.P. provided clinical information and diagnosis. J.K. and Y.H.K. revised the manuscript. All authors reviewed the results and approved the final version of the manuscript.

\section{Competing interests}

The authors declare no competing interests.

\section{Additional information}

Supplementary Information The online version contains supplementary material available at https://doi.org/ 10.1038/s41598-021-97117-6.

Correspondence and requests for materials should be addressed to J.K. or C.J.

Reprints and permissions information is available at www.nature.com/reprints.

Publisher's note Springer Nature remains neutral with regard to jurisdictional claims in published maps and institutional affiliations. 
(c) (i) Open Access This article is licensed under a Creative Commons Attribution 4.0 International cc) License, which permits use, sharing, adaptation, distribution and reproduction in any medium or format, as long as you give appropriate credit to the original author(s) and the source, provide a link to the Creative Commons licence, and indicate if changes were made. The images or other third party material in this article are included in the article's Creative Commons licence, unless indicated otherwise in a credit line to the material. If material is not included in the article's Creative Commons licence and your intended use is not permitted by statutory regulation or exceeds the permitted use, you will need to obtain permission directly from the copyright holder. To view a copy of this licence, visit http://creativecommons.org/licenses/by/4.0/.

(C) The Author(s) 2021 